\title{
Finite Element Method on Shape Memory Alloy Structure and Its Applications
}

\author{
Bo Zhou*, Zetian Kang, Zhiyong Wang and Shifeng Xue
}

\begin{abstract}
It is significant to numerically investigate thermo-mechanical behaviors of shape memory alloy (SMA) structures undergoing large and uneven deformation for they are used in many engineering fields to meet special requirements. To solve the problems of convergence in the numerical simulation on thermo-mechanical behaviors of SMA structures by universal finite element software. This work suppose a finite element method to simulate the super-elasticity and shape memory effect in the SMA structure undergoing large and uneven deformation. Two scalars, named by phase-transition modulus and equivalent stiffness, are defined to make it easy to establish and implement the finite element method for a SMA structure. An incremental constitutive equation is developed to formulate the relationship of stress, strain and temperature in a SMA material based on phase-transition modulus and equivalent stiffness. A phase-transition modulus equation is derived to describe the relationship of phase-transition modulus, stress and temperature in a SMA material during the processes of martensitic phase transition and martensitic inverse phase transition. A finite element equation is established to express the incremental relationship of nodal displacement, external force and temperature change in a finite element discrete structure of SMA. The incremental constitutive equation, phase-transition modulus equation and finite element equation compose the supposed finite element method which simulate the thermo-mechanical behaviors of a SMA structure. Two SMA structures, which undergo large and uneven deformation, are numerically simulated by the supposed finite element method. Results of numerical simulation show that the supposed finite element method can effectively simulate the super-elasticity and shape memory effect of a SMA structure undergoing large and uneven deformation, and is suitable to act as an effective computational tool for the wide applications based on the SMA materials.
\end{abstract}

Keywords: Shape memory alloy, Incremental constitutive equation, Finite element equation, Phase-transition modulus, Applications

\section{Introduction}

Shape memory alloys (SMAs) have been widely used in many various engineering fields [1-4] because they possess two special thermo-mechanical characters, shape memory effect and super-elasticity [5-8]. There are four characteristic temperatures in a SMA at a freestress state. They are named as martensitic starting temperature, indicated by $M_{s}$, martensitic finishing temperature, indicated by $M_{f}$, austenitic starting temperature, indicated by $A_{s}$ and austenitic finishing temperature,

*Correspondence: zhoubo@upc.edu.cn

College of Pipeline and Civil Engineering, China University of Petroleum (East China), Qingdao 266580, China indicated by $A_{f}$ respectively [9-12]. They are generally satisfied with the relationship $M_{f}<M_{s}<A_{s}<A_{f}$

The shape memory effect can be expressed by the stress-strain curve in Figure 1(a), where a large nonlinear strain upon loading becomes a residual strain after unloading at a constant temperature below $A_{s}$. However the residual strain can be fully recovered by heating to a high temperature above $A_{f}$. The super-elasticity can be expressed by the stress-strain curve in Figure 1(b), where the large nonlinear strain upon loading will gradually vanish during the process of unloading at a constant temperature above $A_{f}$ Both shape memory effect and super-elasticity are the macroscopic phenomena of martensitic phase transition and martensitic inverse 


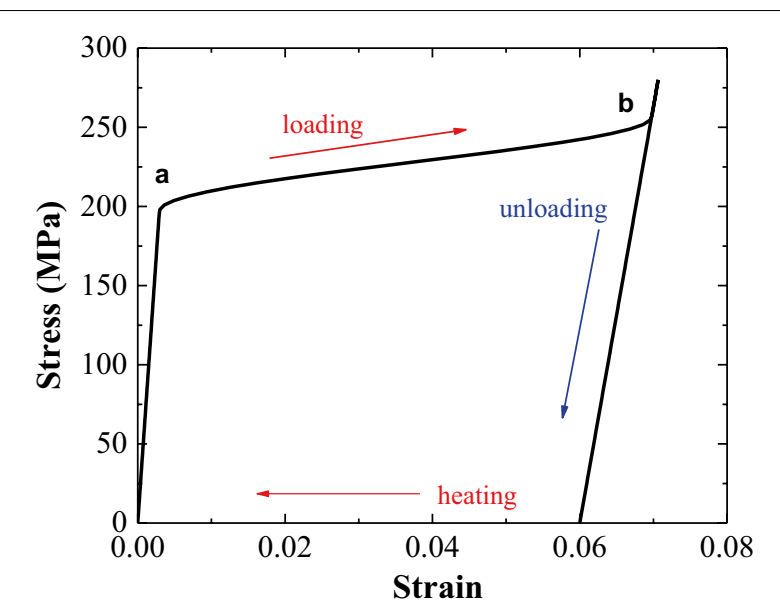

a Shape memory effect

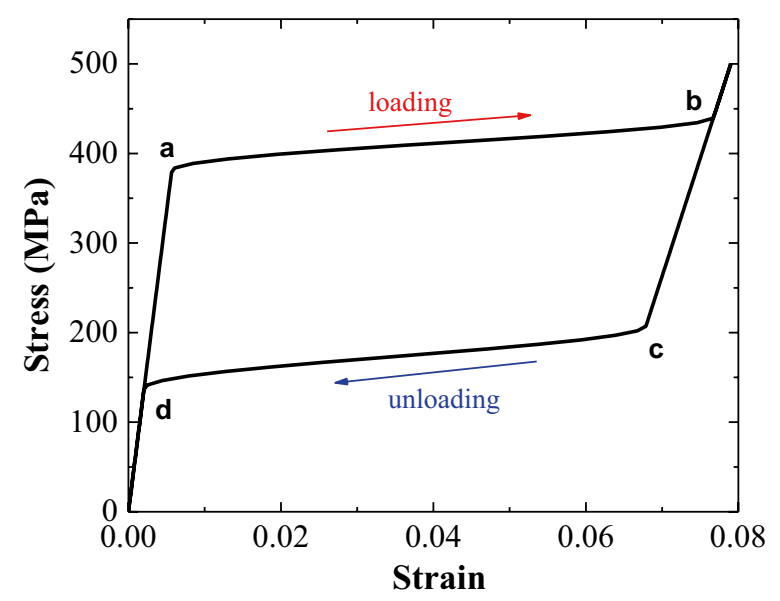

b Super elastic effect

Figure 1 Schematic diagram of shape memory effect and super-elasticity of SMA

phase transition, which are induced by applied stress and temperature change in a SMA material $[13,14]$.

In order to design and analyze a SMA structure efficiently, a numerical simulation method which can effectively predict the thermo-mechanical behaviors of SMA should be established. As a classical numerical computational method, finite element method has been successfully used to solve many problems related to solid and/or fluid mechanics. Many universal finite element software have been successfully applied in different practical fields related to the conventional materials. Some universal finite element software, such as ABAQUS, ANSYS, and so on, have good secondary development functions, which make them can also simulate the problems related to a new materials, such as SMA through calling a user material subroutine. Some researchers [15-17] implemented finite element analysis on SMA structures based on the secondary development functions of ANSYS. Many researchers [18-21] implemented finite element simulation on SMA structures using the secondary development functions of ABAQUS. Although some universal finite element software can simulate the thermo-mechanical behaviors of SMA based on their secondary development functions, the problems of convergence often occur when a SMA structure undergoes large and uneven deformations. However many products or structures made from SMA need undergo large and uneven deformations to meet special requirements. Therefor it is necessary to develop a finite element method and its computer program to simulate the thermo-mechanical behaviors of a SMA structure undergoing large and uneven deformation. Zhou [22] established a finite element program to predict the martensite and plastic zone of SMA thin plate with a hole considering the plastic deformation. However, the super-elasticity and shape memory effect of SMA structures during the thermo-dynamic loading-unloading process haven't been numerically simulated in the work.

To avoid the problems of convergence in the numerical simulation on the thermo-mechanical behaviors of SMA structures with complex boundary conditions and external loads. This work suppose a finite element method to simulate the macroscopic thermo-mechanical responses of super-elasticity and shape memory effect in a SMA structure undergoing large and uneven deformation. In order to make it easy to develop and implement the finite element method for a SMA structure, two scalars named by phase-transition modulus and equivalent stiffness are defined respectively. A concisely incremental constitutive equation formulating the relationship of stress, strain and temperature in a SMA material is derived based on phase-transition modulus and equivalent stiffness. A phase-transition modulus equation is developed to describe the evolution law of phase-transition modulus during the processes of martensitic phase transition and its inverse phase transition in a SMA material. A finite element equation is established to express the incremental relationship of nodal displacement, external force and temperature change in a finite element discrete structure of SMA. The incremental constitutive equation, phasetransition modulus equation and finite element equation compose the element finite method which simulates the thermo-mechanical behaviors of a SMA structure. Two SMA structures are numerically simulated by the supposed finite element method, which illustrates that it can accurately simulate the super-elasticity and shape memory effect in a SMA structure undergoing large and uneven deformation. Therefor the supposed finite 
element method is suitable to act as an effective computational tool for the wide applications based on the SMA materials.

\section{Incremental Constitutive Equation}

Thermo-mechanical constitutive equation of a material is the important basis to investigate the thermo-mechanical behaviors of a SMA material and/or structure. Many constitutive equations have been developed to describe the thermo-mechanical behaviors of SMA material [23-26]. Some constitutive equations from practical viewpoints played important roles in practical applications of SMA [27-30]. In order to make it easy to establish and implement the finite element method for a SMA structure undergoing large and uneven deformation, a concisely incremental constitutive equation should be formulated to describe the thermo-mechanical behaviors of a SMA material.

According to the diagram of shape memory effect and super-elasticity of a SMA, shown in Figure 1, the increment of strain tensor of SMA $\mathrm{d} \varepsilon_{i j}$ can be decomposed as

$$
\mathrm{d} \varepsilon_{i j}=\mathrm{d} \varepsilon_{i j}^{E}+\mathrm{d} \varepsilon_{i j}^{T}+\mathrm{d} \varepsilon_{i j}^{P},
$$

where $\mathrm{d} \varepsilon_{i j}^{E}$, $\mathrm{d} \varepsilon_{i j}^{T}$ and $\mathrm{d} \varepsilon_{i j}^{P}$ stand for the increments of elastic strain tensor, thermal expansion strain tensor and phase-transition strain tensor, respectively.

According to generalized Hooke's law, the increment of stress tensor of SMA d $\sigma_{i j}$ reads as

$$
\mathrm{d} \sigma_{i j}=D_{i j k l} \mathrm{~d} \varepsilon_{k l}^{E},
$$

where $D_{i j k l}$ is the material stiffness tensor. The increment of thermal expansion strain tensor can be expressed as

$$
\mathrm{d} \varepsilon_{i j}^{T}=\Lambda_{i j} \mathrm{~d} T,
$$

where $\Lambda_{i j}$ and $\mathrm{d} T$ are the thermal expansion tensor of a SMA and the increment of temperature respectively.

In this paper a SMA is assumed to be an isotropic material, therefor its stiffness tensor and thermal expansion tensor are formulated as

$$
D_{i j k l}=\frac{E}{1+v}\left[\frac{1}{2}\left(\delta_{i k} \delta_{j l}+\delta_{i l} \delta_{j k}\right)+\frac{v}{1-2 v} \delta_{i j} \delta_{k l}\right],
$$

and

$$
\Lambda_{k l}=\alpha \delta_{k l},
$$

where $E, v$ and $\alpha$ are elastic modulus, Poisson's ratio and thermal expansion coefficient of SMA respectively. The elastic modulus can be expressed as

$$
E=E_{A}-\left(E_{A}-E_{M}\right) \xi
$$

where $\xi, E_{A}$ and $E_{M}$ are martensitic volume fraction, austenitic elastic modulus and martensitic elastic modulus respectively.

Using Eqs. (1), (2) and (3), we can have

$$
\mathrm{d} \sigma_{i j}=D_{i j k l}\left(\mathrm{~d} \varepsilon_{k l}-\Lambda_{k l} \mathrm{~d} T-\mathrm{d} \varepsilon_{k l}^{P}\right) .
$$

In this paper, the increment of phase-transition strain tensor is formulated as

$$
\mathrm{d} \varepsilon_{k l}^{P}=\frac{\partial \bar{\sigma}}{\partial \sigma_{k l}} \mathrm{~d} \bar{\varepsilon}^{P},
$$

where $\bar{\sigma}$ and $\mathrm{d}_{\bar{\varepsilon}}{ }^{P}$ are the equivalent stress and the increment of equivalent phase-transition strain respectively. According to solid mechanics, the equivalent stress reads as

$$
\bar{\sigma}=\sqrt{\frac{3}{2}\left(\sigma_{i j}-\frac{1}{3} \sigma_{k k}\right)\left(\sigma_{i j}-\frac{1}{3} \sigma_{k k}\right)} .
$$

The incremental relationship of equivalent stress and equivalent phase-transition strain can be expressed as

$$
\mathrm{d} \bar{\sigma}=\bar{H} \mathrm{~d} \bar{\varepsilon}^{P},
$$

where $\bar{H}$ is called as phase-transition modulus in this paper. The phase-transition modulus is a scalar which describes the incremental relationship of equivalent stress and equivalent phase-transition strain in a SMA material. The evolution law of phase-transition modulus is derived in Section 3.

On the other hand, the increment of equivalent stress can also be expressed as

$$
\mathrm{d} \bar{\sigma}=\frac{\partial \bar{\sigma}}{\partial \sigma_{i j}} \mathrm{~d} \sigma_{i j}
$$

according to Eq. (7). Using Eqs. (6), (8) and (9), we can have

$$
\mathrm{d} \varepsilon_{k l}^{P}=\frac{\partial \bar{\sigma}}{\partial \sigma_{k l}} \frac{\partial \bar{\sigma}}{\partial \sigma_{i j}} \frac{\mathrm{d} \sigma_{i j}}{\bar{H}} .
$$

From Eqs. (10) and (5), we obtain the incremental constitutive equation of a SMA, expressed as

$$
\mathrm{d} \sigma_{i j}=C_{i j k l} \mathrm{~d} \varepsilon_{k l}-L_{i j} \mathrm{~d} T,
$$

where

$$
\begin{aligned}
& C_{i j k l}=\frac{\bar{H}}{\bar{H}+\bar{D}} D_{i j k l}, \\
& L_{i j}=\frac{\bar{H}}{\bar{H}+\bar{D}} D_{i j k l} \Lambda_{k l} .
\end{aligned}
$$

In Eq. (12), 


$$
\bar{D}=D_{i j k l} \frac{\partial \bar{\sigma}}{\partial \sigma_{k l}} \frac{\partial \bar{\sigma}}{\partial \sigma_{i j}}
$$

is called as equivalent stiffness in this paper. The equivalent stiffness is a scalar which depends on the stiffness tensor and stress state of a material.

The incremental constitutive equation, Eq. (11), is more conveniently used to establish and implement the finite element method for a SMA structure than those constitutive equations of SMA mentioned above, which is due to the definition of phase-transition modulus and equivalent stiffness. During the process of numerical simulation, the values of phase-transition modulus and equivalent stiffness can be calculated according to the current stress state by Eq. (12), and then the increment of stress can be calculated according to the increments of strain and temperature by Eq. (11).

\section{Phase-Transition Modulus Equation}

Based on the cosine-type martensitic phase-transition equation [9], the relationship between martensitic volume fraction and equivalent stress can be expressed as

$$
\xi=\frac{1}{2} \cos \left[\varphi_{1}(\bar{\sigma})\right]+\frac{1}{2},
$$

and

$$
\xi=\frac{1}{2} \cos \left[\varphi_{2}(\bar{\sigma})\right]+\frac{1}{2},
$$

during the processes of martensitic phase transition and martensitic inverse phase transition, respectively.

In Eq. (14a),

$$
\varphi_{1}(\bar{\sigma})=\frac{\bar{\sigma}-\sigma_{m f}}{\sigma_{m s}-\sigma_{m f}} \cdot \pi,
$$

where $\sigma_{m s}$ and $\sigma_{m f}$ are called as martensitic starting stress and martensitic finishing stress respectively. They can be expressed by the martensitic starting temperature and martensitic finishing temperature as

$$
\left\{\begin{array}{c}
\sigma_{m s}=C_{M}\left(T-M_{s}\right), \\
\sigma_{m f}=C_{M}\left(T-M_{f}\right),
\end{array}\right.
$$

where $C_{M}$ is a material constant describing the relationship of stress and temperature during the process of martensitic phase transition in a SMA material.

In Eq. (14b),

$$
\varphi_{2}(\bar{\sigma})=\frac{\bar{\sigma}-\sigma_{a s}}{\sigma_{a f}-\sigma_{a s}} \cdot \pi,
$$

where $\sigma_{a s}$ and $\sigma_{a f}$ are called as austenitic starting stress and austenitic finishing stress respectively. They can be expressed by the austenitic starting temperature and austenitic finishing temperature as

$$
\left\{\begin{array}{l}
\sigma_{a s}=C_{A}\left(T-A_{s}\right), \\
\sigma_{a f}=C_{A}\left(T-A_{f}\right),
\end{array}\right.
$$

where $C_{A}$ is a material constant describing the relationship of stress and temperature during the process of martensitic inverse phase transition in a SMA material.

The incremental relationship of the equivalent phasetransition strain and the martensitic volume fraction can be expressed as

$$
\mathrm{d} \bar{\varepsilon}^{P}=\varepsilon_{L} \mathrm{~d} \xi .
$$

According to Eqs. (8) and (16), we can have

$$
\bar{H}=\left[\varepsilon_{L} \frac{\mathrm{d} \xi}{\mathrm{d} \bar{\sigma}}\right]^{-1} .
$$

Substituting Eq. (14a) into Eq. (17), we can express the phase-transition modulus as

$$
\bar{H}=-\frac{2\left(\sigma_{m s}-\sigma_{m f}\right)}{\varepsilon_{L} \sin \left[\varphi_{1}(\bar{\sigma})\right]},
$$

during the process of martensitic phase transition in a SMA material. Substituting Eq. (14b) into Eq. (17), we can express the phase-transition modulus as

$$
\bar{H}=-\frac{2\left(\sigma_{a f}-\sigma_{a s}\right)}{\varepsilon_{L} \sin \left[\varphi_{21}(\bar{\sigma})\right]},
$$

during the process of martensitic inverse phase transition in a SMA material.

Eq. (18) is the phase-transition modulus equation expressing the evolution law of phase-transition modulus, i.e., the relationship of phase-transition modulus, stress and temperature, during the processes of martensitic phase-transition and martensitic inverse phase-transition occurring in a SMA material.

\section{Finite Element Equation}

According to the stress-strain curves of SMA, shown in Figure 1, both super-elasticity and shape memory effect of a SMA belong to the problem of large deformation. Therefor the Total-Lagrange method suitable for the problem of large deformation is used to develop the finite element equation for a SMA structure. Considering one point in a loaded body, the displacement, GreenLagrange strain and Piola-Kirchhoff stress at the time $t$ and $t+\Delta t$ are respectively expressed as

$$
\begin{gathered}
\quad u_{i}, \varepsilon_{i j}, \sigma_{i j} \\
\text { and } u_{i}+\Delta u_{i}, \varepsilon_{i j}+\Delta \varepsilon_{i j}, \sigma_{i j}+\Delta \sigma_{i j} .
\end{gathered}
$$

They can also be respectively expressed as 


$$
\boldsymbol{u}, \boldsymbol{\varepsilon}, \boldsymbol{\sigma}
$$

and

$$
\boldsymbol{u}+\Delta \boldsymbol{u}, \boldsymbol{\varepsilon}+\Delta \boldsymbol{\varepsilon}, \boldsymbol{\sigma}+\Delta \boldsymbol{\sigma}
$$

by the form of matrix. The increment of strain can be expressed as

$$
\begin{array}{r}
\Delta \varepsilon_{i j}=\frac{1}{2}\left[\Delta u_{j, i}+\Delta u_{i, j}+u_{k, i} \Delta u_{k, j}+\right. \\
\left.\Delta u_{k, i} u_{k, j}+\Delta u_{k, i} \Delta u_{k, j}\right],
\end{array}
$$

which is also expressed as

$$
\Delta \varepsilon=\Delta \varepsilon_{L 0}+\Delta \varepsilon_{L 1}+\Delta \varepsilon_{N}
$$

by the form of matrix, where $\Delta \boldsymbol{\varepsilon}_{L 0}$ is corresponding with the first and second terms, $\Delta \varepsilon_{L 1}$ is corresponding with the third and fourth terms, and $\Delta \varepsilon_{N}$ is corresponding with the fifth term in the right hand of equality sign in Eq. (20) respectively.

At the time $t+\Delta t$, the virtual work equation of a finite element discrete structure reads as

$$
\begin{aligned}
& \sum_{e} \int_{V_{e}} \delta \Delta \boldsymbol{\varepsilon}^{\mathrm{T}}(\boldsymbol{\sigma}+\Delta \boldsymbol{\sigma}) \mathrm{d} V_{e}= \\
& \sum_{e} \int_{V_{e}} \delta \Delta \boldsymbol{u}^{\mathrm{T}} \boldsymbol{f} \mathrm{d} V_{e}+\sum_{e} \int_{S_{e}} \delta \Delta \boldsymbol{u}^{\mathrm{T}} \boldsymbol{q} \mathrm{d} S_{e},
\end{aligned}
$$

where $\delta$ is the symbol of variation, $V_{e}$ and $S_{e}$ express the volume and surface of an element, and $\boldsymbol{f}$ and $\boldsymbol{q}$ stand for body force array and surface force array respectively. The increment of displacement array at one point in an element can be expressed by the increment of nodal displacement array of the element as

$$
\Delta \boldsymbol{u}=N \Delta \boldsymbol{u}^{e},
$$

where $\Delta \boldsymbol{u}^{e}$ is the increment of nodal displacement array of the element and $N$ is the shape function matrix of the element, respectively.

Operating the variation operation on Eq. (22) leads to

$$
\delta \Delta \boldsymbol{u}=\boldsymbol{N} \delta \Delta \boldsymbol{u}^{e} .
$$

The increment of strain array is formulated by the increment of nodal displacement array as

$$
\Delta \boldsymbol{\varepsilon}=\boldsymbol{B} \Delta \boldsymbol{u}^{e}=\left(\boldsymbol{B}_{L 0}+\boldsymbol{B}_{L 1}+\boldsymbol{B}_{N}\right) \Delta \boldsymbol{u}^{e},
$$

where $\boldsymbol{B}$ is the strain matrix of element. It includes $\boldsymbol{B}_{L 0}$, $\boldsymbol{B}_{L 1}$ and $\boldsymbol{B}_{N}$, which are with respect to the $\Delta \boldsymbol{\varepsilon}_{L 0}, \Delta \boldsymbol{\varepsilon}_{L 1}$ and $\Delta \boldsymbol{\varepsilon}_{N}$ in Eq. (20), respectively. According to Eqs. (19) and (24), if the higher order small term is ignored, the variation of the increment of strain can be expressed as

$$
\delta \Delta \boldsymbol{\varepsilon}=\boldsymbol{B} \delta \Delta \boldsymbol{u}^{e}=\left(\boldsymbol{B}_{L 0}+\boldsymbol{B}_{L 1}\right) \delta \Delta \boldsymbol{u}^{e} .
$$

In order to establish finite element equation for a SMA structure, we express the incremental constitutive equation, Eq. (11), with the form of matrix as

$$
\Delta \boldsymbol{\sigma}=\boldsymbol{C} \Delta \boldsymbol{\varepsilon}-\boldsymbol{L} \Delta T .
$$

Substituting Eqs. (22)-(26) into Eq. (21) and carrying out the necessary derivations and reductions, we can have

$$
\begin{aligned}
-\delta \Delta & \boldsymbol{U}^{\mathrm{T}} \boldsymbol{P}_{\sigma}+\delta \Delta \boldsymbol{U}^{\mathrm{T}} \boldsymbol{K}_{L} \Delta \boldsymbol{U}+ \\
& \delta \Delta \boldsymbol{U}^{\mathrm{T}} \boldsymbol{K}_{N} \Delta \boldsymbol{U}-\delta \Delta \boldsymbol{U}^{\mathrm{T}} \boldsymbol{P}_{T}=\delta \Delta \boldsymbol{U}^{\mathrm{T}} \boldsymbol{P}_{F},
\end{aligned}
$$

where

$$
\delta \Delta \boldsymbol{U}^{\mathrm{T}} \boldsymbol{P}_{\sigma}=-\sum_{e} \int_{V_{e}} \delta \Delta \boldsymbol{u}^{e \mathrm{~T}}\left(\boldsymbol{B}_{L 0}^{\mathrm{T}}+\boldsymbol{B}_{L 1}^{\mathrm{T}}\right) \boldsymbol{\sigma} \mathrm{d} V_{e},
$$

$$
\begin{gathered}
\delta \Delta \boldsymbol{U}^{\mathrm{T}} \boldsymbol{K}_{L} \Delta \boldsymbol{U}=\sum_{e} \int_{V} \delta \Delta \boldsymbol{u}^{e \mathrm{~T}} \times \\
\left(\boldsymbol{B}_{L 0}^{\mathrm{T}}+\boldsymbol{B}_{L 1}^{\mathrm{T}}\right) \boldsymbol{C}\left(\boldsymbol{B}_{L 0}+\boldsymbol{B}_{L 1}\right) \Delta \boldsymbol{u}^{e} \mathrm{~d} V_{e},
\end{gathered}
$$

$$
\begin{array}{r}
\delta \Delta \boldsymbol{U}^{\mathrm{T}} \boldsymbol{K}_{N} \Delta \boldsymbol{U}=\sum_{e} \int_{V} \delta \Delta \boldsymbol{u}^{e \mathrm{~T}} \times \\
\left(\boldsymbol{B}_{L 0}^{\mathrm{T}}+\boldsymbol{B}_{L 1}^{\mathrm{T}}\right) \boldsymbol{C} \boldsymbol{B}_{N} \Delta \boldsymbol{u}^{e} \mathrm{~d} V_{e}
\end{array}
$$

$$
\delta \Delta \boldsymbol{U}^{\mathrm{T}} \boldsymbol{P}_{T}=\sum_{e} \int_{V_{e}} \delta \Delta \boldsymbol{u}^{e \mathrm{~T}}\left(\boldsymbol{B}_{L 0}^{\mathrm{T}}+\boldsymbol{B}_{L 1}^{\mathrm{T}}\right) \boldsymbol{L} \Delta T \mathrm{~d} V_{e}
$$

$$
\begin{aligned}
\delta \Delta \boldsymbol{U}^{\mathrm{T}} \boldsymbol{P}_{F} & =\sum_{e} \int_{V_{e}} \delta \Delta \boldsymbol{u}^{e \mathrm{~T}} \boldsymbol{N}^{\mathrm{T}} \boldsymbol{f} \mathrm{d} V_{e} \\
& +\sum_{e} \int_{S_{e}} \delta \Delta \boldsymbol{u}^{e \mathrm{~T}} \boldsymbol{N}^{\mathrm{T}} \boldsymbol{q} \mathrm{d} S_{e} .
\end{aligned}
$$

The $\Delta \boldsymbol{U}$ and $\delta \Delta \boldsymbol{U}$ in Eq. (28) denote the increment of nodal displacement array and its virtual displacement array of the finite element discrete structure respectively. The $\boldsymbol{P}_{F}$ in Eq. (28e) is the equivalent nodal loads array reduced from external force. The $\boldsymbol{P}_{T}$ in Eq. (28d) is the equivalent nodal loads array reduced from the change of temperature. The $\boldsymbol{P}_{\sigma}$ in Eq. (28a) is the equivalent nodal loads array reduced from non-equilibrium force.

The nodal virtual displacement array $\delta \Delta \boldsymbol{U}$ should be arbitrary, so Eq. (27) can be arranged and reduced as

$$
\left(\boldsymbol{K}_{L}+\boldsymbol{K}_{N}\right) \Delta \boldsymbol{U}=\boldsymbol{P}_{F}+\boldsymbol{P}_{T}+\boldsymbol{P}_{\sigma},
$$

where $\boldsymbol{K}_{L}$ and $\boldsymbol{K}_{N}$ express structural stiffness matrices which relate with small deformation and large 


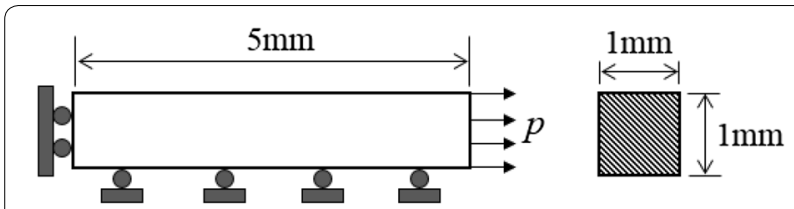

a A SMA tensile bar

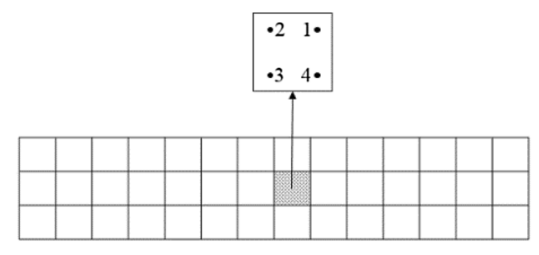

b Finite element mesh of SMA bar

Figure 2 A SMA tensile bar and its finite element mesh

deformation respectively. The nonlinear equation, Eq. (29), is the finite element equation which describes the relationship of the nodal displacement, external force and temperature change in a finite element discrete structure of SMA. This non-linear equation is solved by the Newton-Raphson method during the processes of finite element simulations on the SMA structures in this paper.

The incremental constitutive equation, Eq. (11), the phase-transition modulus equation, Eq. (18), and the finite element equation, Eq. (29), compose the supposed finite element method, which simulate the thermomechanical macroscopic response of super-elasticity and shape memory effect occurring in a SMA structure.

\section{Applications}

In order to operate finite element simulations on the thermo-mechanical response of a SMA structure using the supposed finite element method mentioned above, a MATLAB program is compiled based on finite element equation, Eq. (29), incremental constitutive equation, Eq. (11), and phase-transition modulus equation, Eq. (18). A uniform tensile SMA bar, shown in Figure 2(a), is numerically simulated to verify the MATLAB program. The material parameters of SMA [23] used for the numerical simulations are listed in Table 1. Figure 2(b) plots the finite element mesh of the SMA bar, which include 42 plane strain four-node elements. The numerical results at integral point 1 of the shaded element, shown in Figure 2(b), are used to compare with the analytic solutions [9] to illustrate the validity of the supposed finite element method in this paper.

Figure 3(a) plots the stress-strain curve of the integral point 1, shown in Figure 2(b), at a constant temperature above austenitic finishing temperature $A_{f}$. The large nonlinear phase-transition strain produced in the loading
Table 1 Material parameters of SMA [23]

\begin{tabular}{llll}
\hline$M_{f}(\mathrm{~K})$ & $M_{s}(\mathrm{~K})$ & $A_{s}(\mathrm{~K})$ & $A_{f}(\mathrm{~K})$ \\
271 & 291 & 295 & 315 \\
$E_{A}(\mathrm{GPa})$ & $E_{M}(\mathrm{GPa})$ & $C_{A}(\mathrm{MPa} / \mathrm{K})$ & $C_{M}(\mathrm{MPa} / \mathrm{K})$ \\
70 & 30 & 7.0 & 7.0 \\
$\varepsilon_{L}$ & $V$ & $a(1 / \mathrm{K})$ & \\
0.06 & 0.33 & $1.0 \times 10^{-7}$ & \\
\hline
\end{tabular}

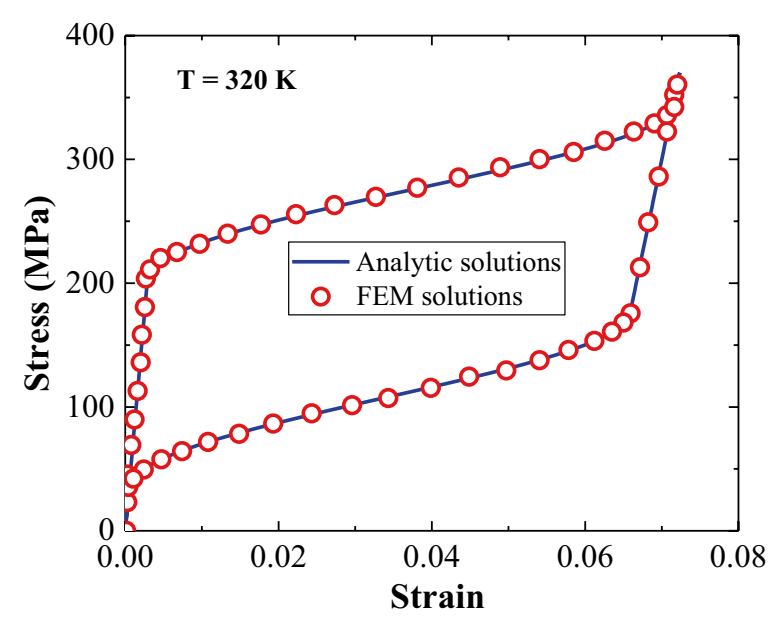

a The stress-strain curves at $320 \mathrm{~K}$

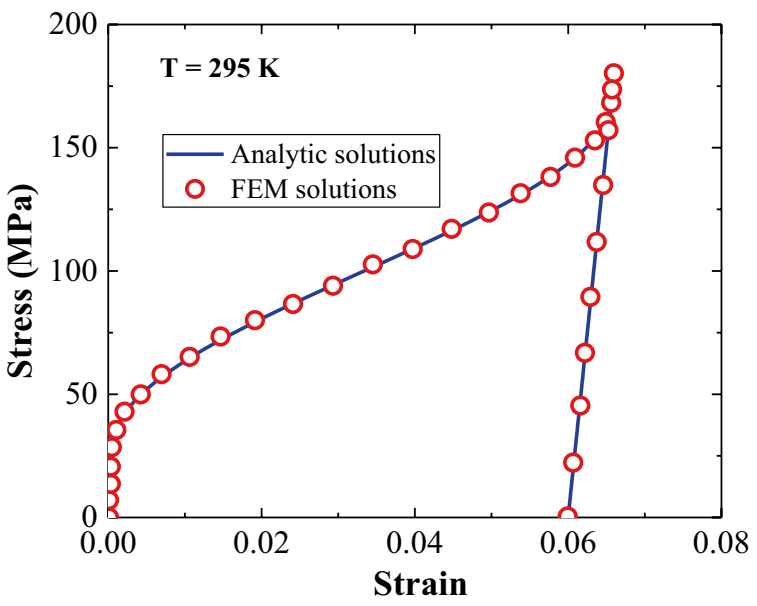

b The stress-strain curves at $295 \mathrm{~K}$

Figure 3 Stress-strain curves of SMA at various constant temperatures

process is fully recovered during the unloading process, which illustrates the super-elasticity of a SMA material. Figure 3(b) shows the stress-strain curve of the integral point 1, shown in Figure 2(b), at a constant temperature below austenitic finishing temperature $A_{f}$ The large nonlinear phase-transition strain produced in the loading 
process becomes residual strain after the unloading process. The residual strain can be fully recovered upon heating to a high temperature above $A_{f}$ which illustrates the shape memory effect of a SMA material.

According to the curves in Figure 3(a) and Figure 3(b), the results from the supposed finite element method in this paper have a good agreement with that from the analytic solutions [9]. Therefore the supposed finite element method in this paper can effectively simulate the thermomechanical macroscopic responses of super-elasticity and shape memory effect occurring in a SMA structure.

\subsection{Thermo-mechanical Behaviors of SMA Bar}

Figure 4 shows a deformation mechanism for a SMA bar, which includes two punch heads, one die and one support. The moments of force applied on the punch head make the punch head rotate around the rotation point and generate a large and uneven bending deformation in the SMA bar. The SMA bar has length of $28 \mathrm{~mm}$, width of $2 \mathrm{~mm}$ and thickness of $4 \mathrm{~mm}$. The radius of arc in the punch head is $6 \mathrm{~mm}$, and the radius of the head in the die is $8 \mathrm{~mm}$. During the numerical simulations the SMA bar is meshed by the 4-node plane strain element, and the total number of element is 224 . The punch head and die are assumed to be rigid body. The friction-free contacts are set between punch head and SMA bar and between punch head and SMA bar. The material constants of SMA bar are listed in Table 1.

Figure 5 plots the relational curve between the moment of force and the rotation angle of punch head at a constant temperature of $317 \mathrm{~K}$. During the loading process there is a nonlinear curve segment occurring, which is the results of martensitic phase transition occurring in some materials of the SMA bar. During the unloading process there is a non-linear curve segment occurring, which is the result of martensitic inverse phase transition

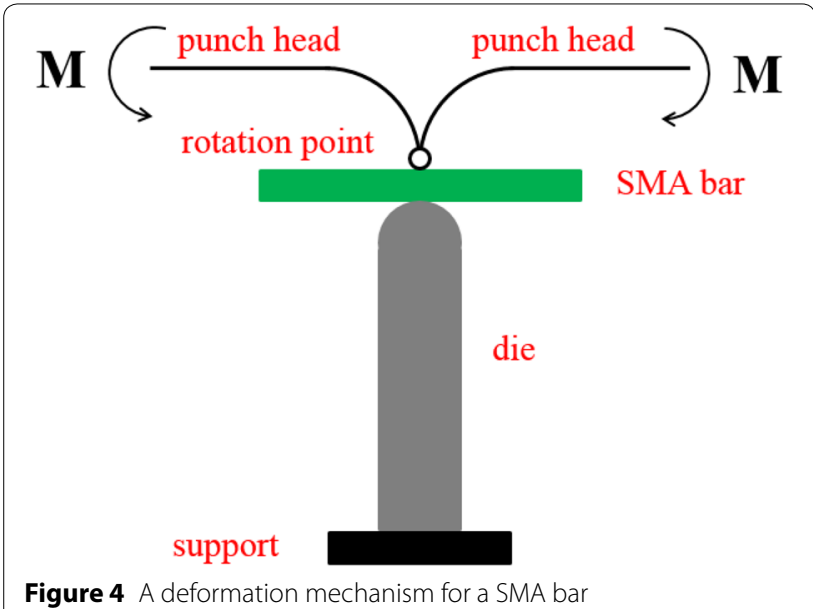

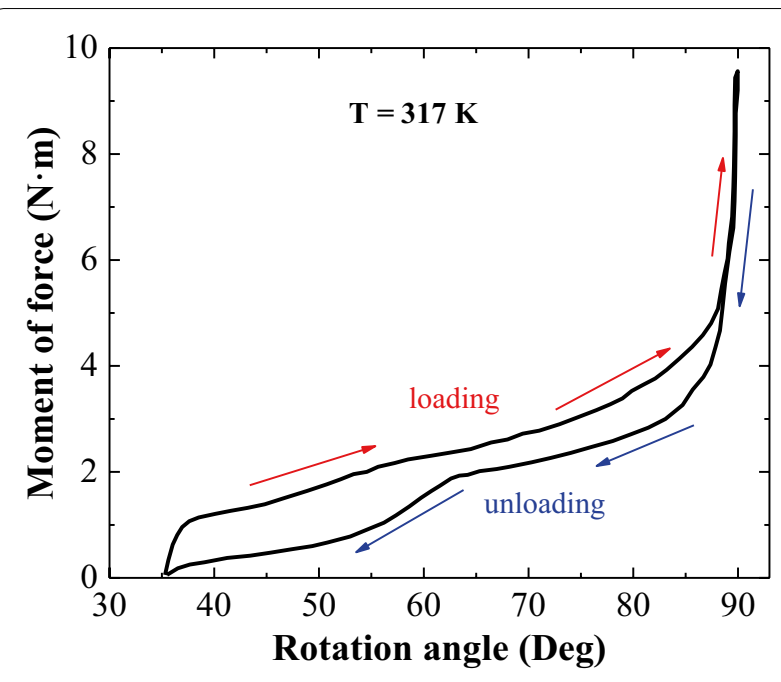

Figure 5 Curve of the moment of force versus the rotation angle of punch head at $317 \mathrm{~K}$

occurring in the materials having experienced martensitic phase transition in the loading process. There is a hysteresis loop occurring in the curve, which illustrates the super-elasticity of the SMA bar. Figure 6 plots the relational curve between the moment of force and the rotation angle of punch head at a constant temperature of $300 \mathrm{~K}$. During the loading process there is also a nonlinear curve segment occurring, which is also the result of martensitic phase transition occurring in some materials of SMA bar. However there is not a hysteresis loop occurring in the curve after the unloading process.

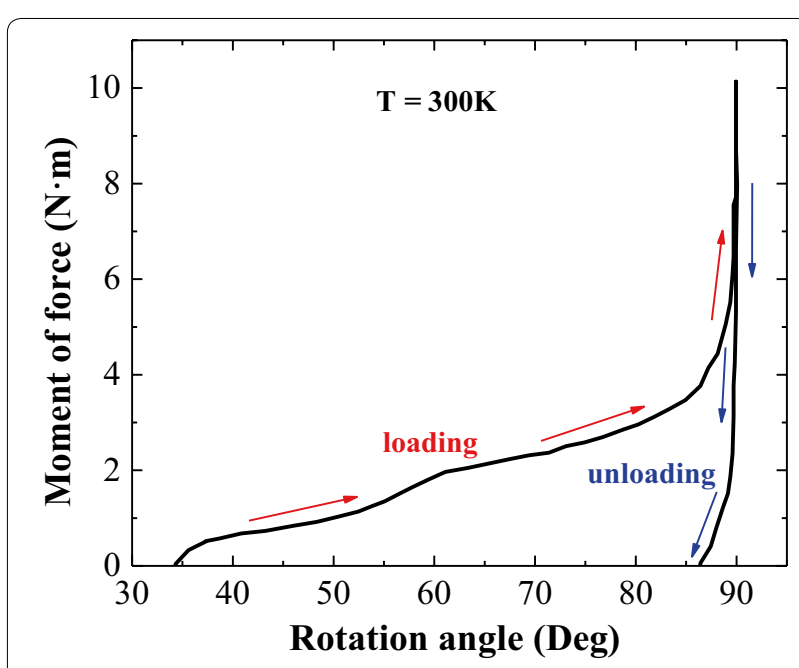

Figure 6 Curve of the moment of force versus the rotation angle of punch head at $300 \mathrm{~K}$ 

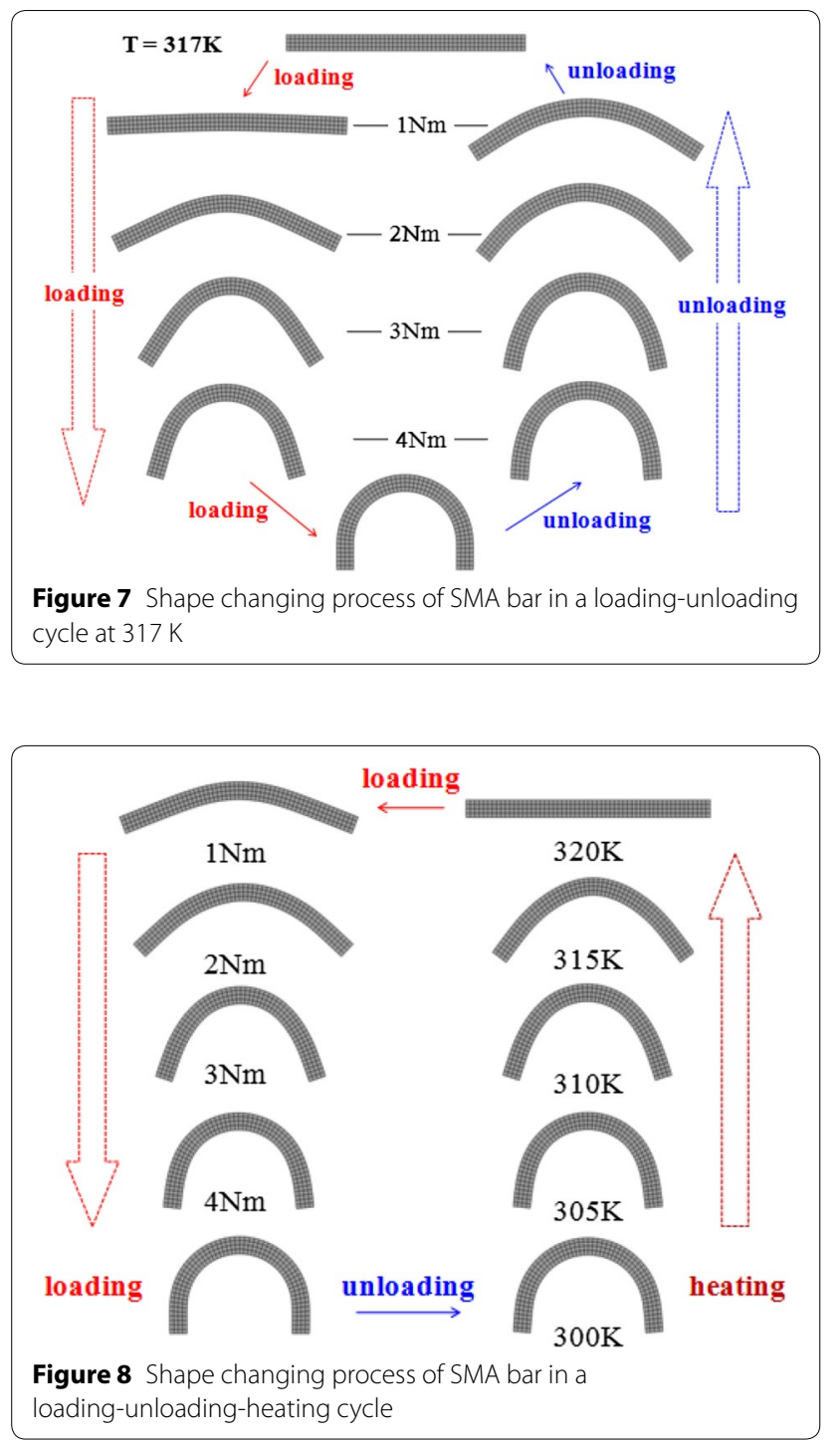

Figure 7 shows the shape changing process of SMA bar at a constant temperature of $317 \mathrm{~K}$ in the loadingunloading cycle. The deformed shapes of SMA bar at the same moment of force during the processes of loading and unloading are different. This is because the loadingpath does not coincide with the unloading-path, which is results from the hysteresis loop shown in Figure 5. Figure 8 shows the shape changing process of SMA bar in the loading-unloading-heating cycle. The deformation of SMA bar, which is induced by the martensitic phase transition upon loading, becomes the residual deformation after the unloading process. The residual deformation in the SMA bar is fully recovered by heating it from $300 \mathrm{~K}$ to $320 \mathrm{~K}$, which illustrates the shape memory effect of SMA bar.
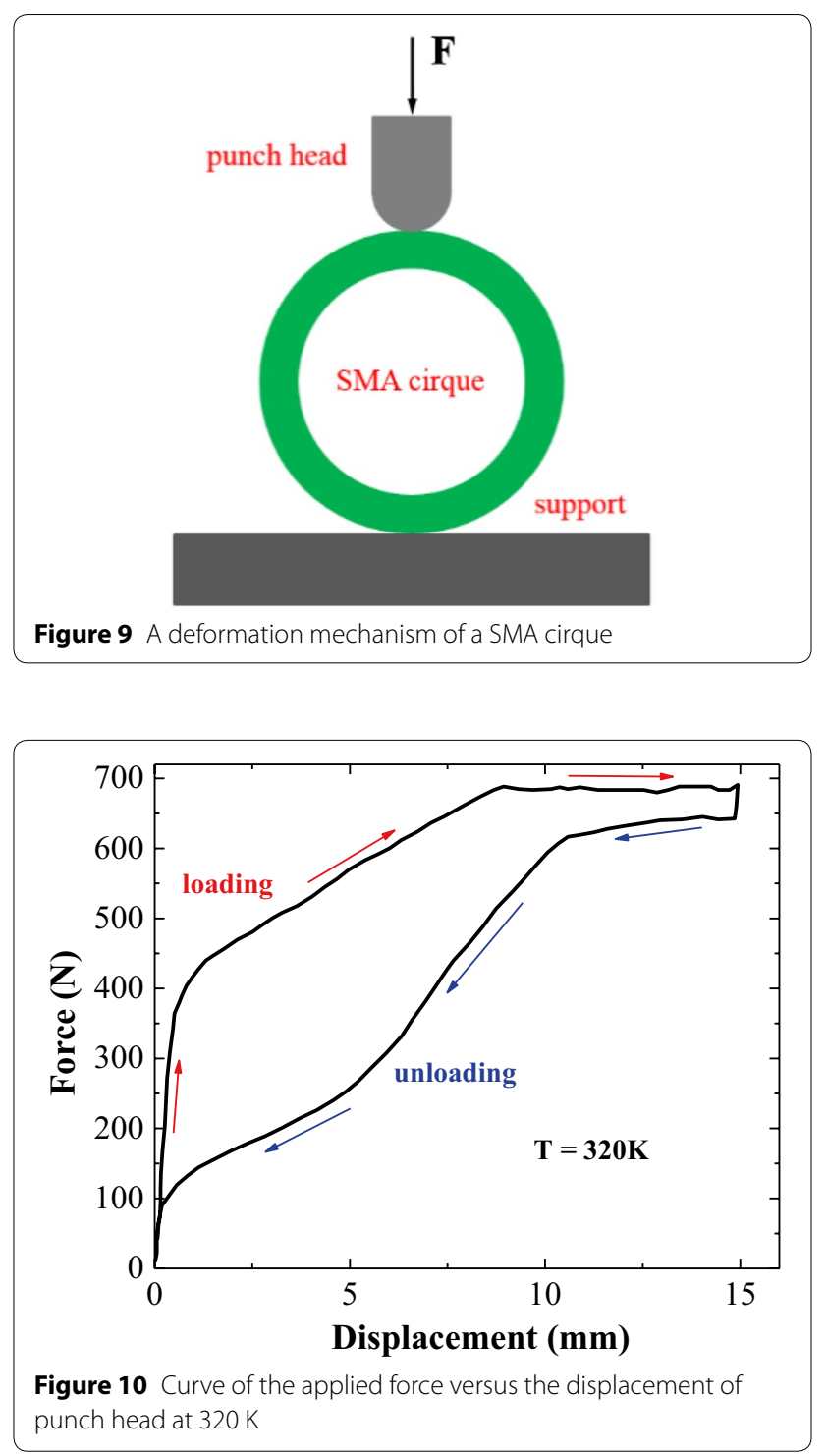

\subsection{Thermo-mechanical Behaviors of SMA Cirque}

Figure 9 shows a deformation mechanism for a SMA cirque, which includes a punch head and a support. The applied force makes the punch head move down and produce large and uneven deformation in the SMA cirque. The radius of punch head bottom is $2 \mathrm{~mm}$. The inside radius and outer radius of SMA cirque are $10 \mathrm{~mm}$ and $12 \mathrm{~mm}$ respectively. The thickness of SMA cirque is $4 \mathrm{~mm}$. During the numerical simulations the SMA cirque is meshed by the 4-node plane strain element, and the total number of element is 544 . The punch head is assumed to be a rigid body. The friction-free contacts are set between the punch head and SMA cirque. The material parameters of SMA cirque are listed in Table 1. 
Figure 10 plots the relational curve between the applied force and the displacement of punch head at a constant temperature of $320 \mathrm{~K}$. During the loading process there is a nonlinear curve segment due to the martensitic phase transition occurring in some materials of the SMA cirque. During the unloading process there is also a nonlinear curve segment occurring, which is the result of martensitic inverse phase transition occurring in the materials having experienced martensitic phase transition in the loading process. There is a hysteresis loop occurring in the curve after unloading, which illustrates the super-elasticity of SMA cirque. Figure 11 plots the relational curve between the applied force and the displacement of punch head at a constant temperature of $295 \mathrm{~K}$. During the loading process there is a nonlinear curve segment due to the martensitic phase transition occurring in some materials of SMA cirque. There is not a hysteresis loop occurring in the curve after unloading.

Figure 12 shows the shape changing process of SMA cirque in a loading-unloading cycle at a constant temperature of $320 \mathrm{~K}$. The deformed shapes of SMA cirque corresponding with the same applied force during the processes of loading and unloading are different because the loading-path does not coincide with the unloading-path, as shown in Figure 10. Figure 13 shows the shape changing process of SMA cirque in the loading-unloading-heating cycle. The deformation of SMA cirque, which is induced by the martensitic phase transition upon loading, becomes the residual deformation after unloading. However, the residual deformation in the SMA cirque is fully recovered by heating it from

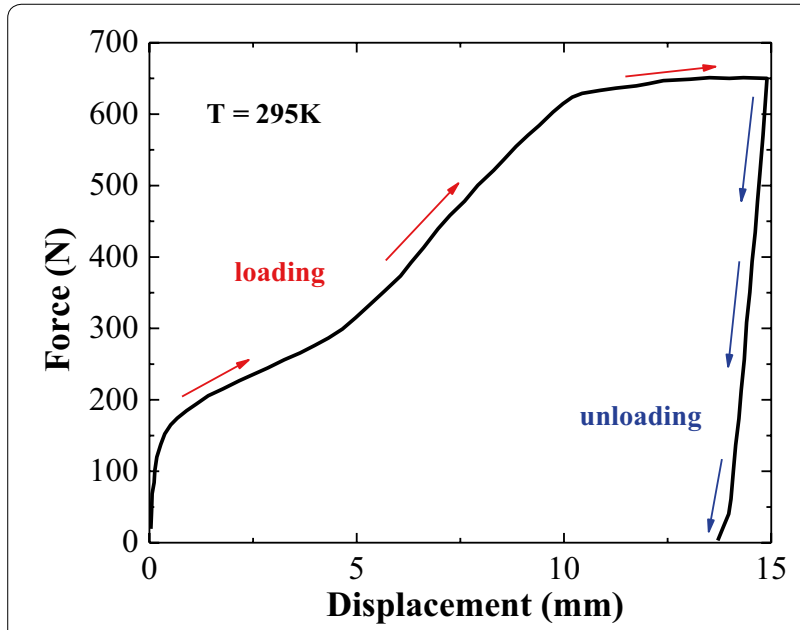

Figure 11 Curve of the applied force versus the displacement of punch head at $295 \mathrm{~K}$

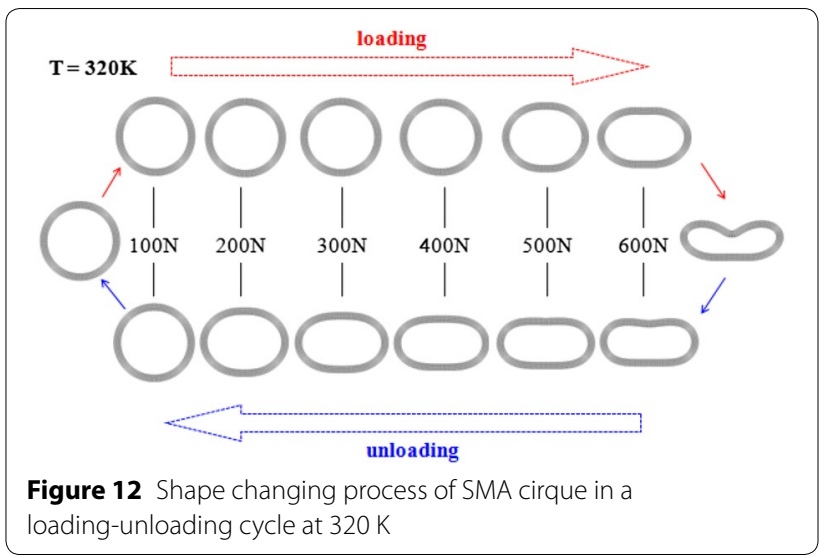

$295 \mathrm{~K}$ to $320 \mathrm{~K}$, which illustrates the shape memory effect of SMA cirque.

On the whole, the supposed finite element method has good accuracy and convergence in the simulation on both super-elasticity and shape memory effect in a SMA structure, and is suitable to be an effective computational tool for the wide applications based on a SMA material.

\section{Conclusions}

Two scalars, phase-transition modulus and equivalent stiffness, are defined to establish and implement the finite element method. The super-elasticity and shape memory effect of SMA bar and cirque are respectively simulated by the supposed finite element method. Accordingly, several conclusions are presented as follows.

1) The concisely incremental constitutive equation describing the relationship of stress, strain and temperature in a SMA material is developed based on phase-transition modulus and equivalent stiffness.

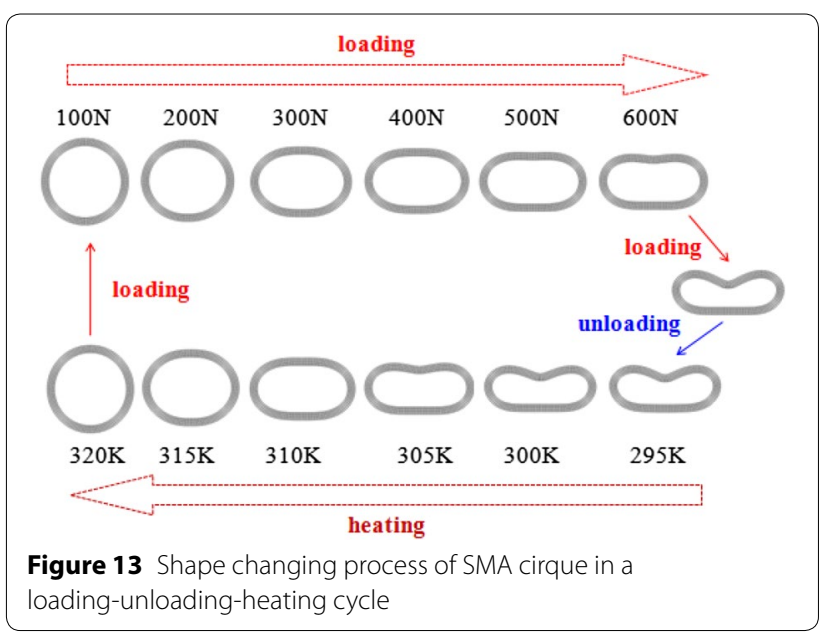


2) The phase-transition modulus equation expressing the relationship of phase-transition modulus, stress and temperature during the processes of martensitic phase transition and martensitic inverse phase transition in a SMA material is presented.

3) The finite element equation formulating the incremental relationship of nodal displacement, external force and temperature change in a finite element discrete structure is established to simulate the thermomechanical behaviors of a SMA structure.

4) The supposed finite element method, which includes the incremental constitutive equation, phase-transition modulus equation and finite element equation, can effectively simulate both processes of super-elasticity and shape memory effect in a SMA structure, and is suitable to act as an effective computational tool for the wide applications based on a SMA material.

\section{Authors' Contributions}

BZ was in charge of the whole trial; ZK and ZW compiled the calculation programs and wrote Section 5; SX wrote Section 4. All authors read and approved the final manuscript.

\section{Authors' Information}

Bo Zhou, born in 1972, is currently a professor and a doctoral supervisor at Department of Engineering Mechanics, College of Pipeline and Civil Engineering, China University of Petroleum, China. His research interests include intelligent materials and structural mechanics, computational mechanics and engineering mechanics of oil and gas wells.

Zetian Kang, born in 1992, is currently a PhD candidate at China University of Petroleum, China. He received his bachelor degree from China University of Petroleum, in 2014. His research interest is intelligent materials and structural mechanics.

Zhiyong Wang, born in 1992, is currently a graduate student at China University of Petroleum, China. He received his bachelor degree from China University of Petroleum, China, in 2017. His research interest is intelligent materials and structural mechanics.

Shifeng Xue, born in 1963, is currently a professor and a doctoral supervisor at Department of Engineering Mechanics, College of Pipeline and Civil Engineering, China University of Petroleum, China. His research interests include computational mechanics, fracture mechanics and engineering mechanics of oil and gas wells.

\section{Acknowledgements}

The authors of this paper sincerely thank to the supports from the China University of Petroleum (East China).

\section{Competing Interests}

The authors declare that they have no competing interests.

\section{Funding}

Supported by National Key Research and Development Program of China (Grant No. 2017YFC0307604), and the Talent Foundation of China University of Petroleum (Grant No. Y1215042).

Received: 5 December 2018 Revised: 3 June 2019 Accepted: 14 October 2019

Published online: 24 October 2019

\section{References}

[1] N Choudhary, D Kaur. Shape memory alloy thin films and heterostructures for MEMS applications: A review. Sensors and Actuators A: Physical, 2016, 242: 162-181. https://doi.org/10.1016/j.sna.2016.02.026

[2] Y Zheng, Y Dong, Y H Li. Resilience and life-cycle performance of smart bridges with shape memory alloy (SMA)-cable-based bearings. Construction and Building Materials, 2018, 158: 389-400. https://doi.org/10.1016/j. conbuildmat.2017.10.031

[3] WT Jhou, CWang, S Li, et al. TiNiCuAg shape memory alloy films for biomedical applications. Journal of Alloys and Compounds, 2018, 738: 336344. https://doi.org/10.1016/j.jallcom.2017.12.194

[4] P B C Leal, M A Savi. Shape memory alloy-based mechanism for aeronautical application: Theory, optimization and experiment. Aerospace Science and Technology, 2018, 76: 155-163. https://doi.org/10.1016/j. ast.2018.02.010

[5] B Zhou. A macroscopic constitutive model of shape memory alloy considering plasticity. Mechanics of Materials, 2012, 48: 71-81. https://doi. org/10.1016/j.mechmat.2012.02.001

[6] B Zhou, ZY Wang, S F Xue. Mechanical model for super-elastic helical spring of shape memory alloy. Journal of Mechanical Engineering, 2019, 55(8): 56-64. https://doi.org/10.3901/JME.2019.08.056 (in Chinese)

[7] ZT Kang, B Zhou, S F Xue. Mechanical behaviors of functionally graded shape memory alloy composite beam. Acta Materiae Compositae Sinica, 2019, 36(08): 1901-1910. https://doi.org/10.13801/j.cnki.fhclxb.20181 114.003 (in Chinese)

[8] J Wang, Z Moumni, W H Zhang, et al. A thermomechanically coupled finite deformation constitutive model for shape memory alloys based on Hencky strain. International Journal of Engineering Science, 2017, 117: 51-77. https://doi.org/10.1016/j.ijengsci.2017.05.003

[9] C Liang, C Rogers. One-Dimensional Thermo-mechanical Constitutive Relations for Shape Memory Materials. Journal of Intelligent Material Systems and Structures, 1990, 35: 207-234. https://doi.org/10.1177/10453 89X9000100205

[10] L C Brinson. One-Dimensional Constitutive Behavior of Shape Memory Alloys: Thermo-mechanical Derivation with Non-Constant Material Functions and Redefined Martensite Internal Variable. Journal of Intelligent Material Systems and Structures, 1993, 4: 229-242. https://doi. org/10.1177/1045389X9300400213

[11] C Cisse, W Zaki, T Ben Zineb. A review of constitutive models and modeling techniques for shape memory alloys. International Journal of Plasticity, 2016, 76: 244-284. https://doi.org/10.1016/j.ijplas.2015.08.006

[12] X Chen, T Liu, R Li, et al. Molecular dynamics simulation on the shape memory effect and superelasticity in NiTi shape memory alloy. Computational Materials Science, 2018, 146: 61-69. https://doi.org/10.1016/j. commatsci.2018.01.026

[13] B Zhou, S Yoon, J S Leng. A three-dimensional constitutive model for shape memory alloy. Smart Materials and Structures, 2009, 18: 9-16. https ://doi.org/10.1088/0964-1726/18/9/095016

[14] B Zhou, Y J Liu, J S Leng, et al. A macro-mechanical constitutive model of shape memory alloys. Science in China, 2009, 52(9): 1382-1391. https://doi. org/10.1007/s11433-009-0173-3

[15] P Terriault, F Viens, V Brailovski. Non-isothermal finite element modeling of a shape memory alloy actuator using ANSYS. Computational Materials Science, 2006, 36: 397-410. https://doi.org/10.1016/j.comma tsci.2005.05.010

[16] H S Lei, Z Q Wang, B Zhou, et al. Simulation and analysis of shape memory alloy fiber rein-forced composite based on cohesive zone model. Materials and Design, 2012, 40: 138-147. https://doi.org/10.1016/j. matdes.2012.03.037

[17] D D Gu, B B He. Finite element simulation and experimental investigation of residual stresses in selective laser melted Ti-Ni shape memory alloy. Computational Materials Science, 2016, 117: 221-232. https://doi. org/10.1016/j.commatsci.2016.01.044

[18] E A P Hernandez, B Kiefer, D J Hartl, et al. Analytical investigation of structurally stable configurations in shape memory alloy-actuated plates. International Journal of Solids and Structures, 2015, 69-70: 442-458. https:// doi.org/10.1016/j.ijsolstr.2015.05.007

[19] K M Armattoe, C Bouby, M Haboussi, et al. Modeling of latent heat effects on phase transformation in shape memory alloy thin structures. International Journal of Solids and Structures, 2016, 88: 283-295. https://doi. org/10.1016/j.ijsolstr.2016.02.024 
[20] X Long, X Peng, T Fu, et al. A micro-macro description for pseudo-elasticity of NiTi SMAs subjected to non-proportional deformations. International Journal of Plasticity, 2017, 90: 44-65. https://doi.org/10.1016/j.ijpla s.2016.12.003

[21] ZT Kang, B Zhou, S F Xue. Finite element numerical simulation on thermo-mechanical coupling behavior in shape memory alloy pipe connection. Journal of Mechanical Engineering, 2018, 54(18): 68-75. https:// doi.org/10.3901/JME.2018.18.068 (in Chinese)

[22] B Zhou. Finite element method of shape memory alloy and its applications. China Mechanics Conference 2017 \& The 60th Anniversary of China Mechanics Society. Beijing: China Academic Journal Electronic Publishing House, 2017. (in Chinese)

[23] D C Lagoudas, Z H Bo. Thermo-mechanical modeling of polycrystalline SMAs under cyclic loading, Part II: Material characterization and experimental results for a stable transformation cycle. International Journal of Engineering Science, 1999, 37: 1141-1173. https://doi.org/10.1016/S0020 -7225(98)00114-1

[24] F Auricchio, E Bonetti, G Scalet, et al. Theoretical and numerical modeling of shape memory alloys accounting for multiple phase transformations and martensite reorientation. International Journal of Plasticity, 2014, 59: 30-54. https://doi.org/10.1016/j.ijplas.2014.03.008

[25] CYu, G Z Kang, Q H Kan. A micromechanical constitutive model for anisotropic cyclic deformation of super-elastic NiTi shape memory alloy single crystals. Journal of the Mechanics and Physics of Solids, 2015, 82: 97-136. https://doi.org/10.1016/j.jmps.2015.05.012
[26] B Wang, G Z Kang, Q H Kan, et al. Molecular dynamics simulations to the pseudo-elasticity of NiTi shape memory alloy nano-pillar subjected to cyclic compression. Computational Materials Science, 2017, 131: 132-138. https://doi.org/10.1016/j.commatsci.2017.01.045

[27] J G Boyd, D C Lagoudas. A thermodynamical constitutive model for shape memory materials. Part I. The monolithic shape memory alloy. International Journal of Plasticity, 1996, 12: 805-842. https://doi. org/10.1016/S0749-6419(96)00030-7

[28] F Auricchio, E Sacco. A one-dimensional model for super-elastic shapememory alloys with different elastic properties between austenite and martensite. International Journal of Non-Linear Mechanics, 1997, 32: 11011114. https://doi.org/10.1016/S0020-7462(96)00130-8

[29] M J Ashrafi, J Arghavani, R Naghdabadi, et al. Theoretical and numerical modeling of dense and porous shape memory alloys accounting for coupling effects of plasticity and transformation. International Journal of Solids and Structures, 2015, 88: 248-262. https://doi.org/10.1016/j.ijsol str.2016.03.003

[30] CYu, G Z Kang, K J Chen, et al. A thermo-mechanically coupled nonlinear viscoelastic-viscoplastic cyclic constitutive model for polymeric materials. Mechanics of Materials, 2017, 105: 1-15. https://doi.org/10.1016/j.mechm at.2016.11.004

\section{Submit your manuscript to a SpringerOpen ${ }^{\circ}$ journal and benefit from:}

- Convenient online submission

- Rigorous peer review

- Open access: articles freely available online

- High visibility within the field

- Retaining the copyright to your article

Submit your next manuscript at $\boldsymbol{\nabla}$ springeropen.com 